\title{
Precoding and Transmitter-Side processing techniques for multiuser MIMO OFDM systems with special emphasis on the PAPR problem
}

\author{
G Wunder $^{1 *}$, RFH Fischer ${ }^{2}, \mathrm{~S}$ Litsyn $^{3}$ and JS No ${ }^{4}$
}

Green information technology has significantly fuelled the discussion about energy efficiency in information networks. Already today, energy cost of network operation can make up as much as $50 \%$ of the total operational cost. Notably, by the complexity of networks in general, the efficiency problem is hard to tackle on a global scale but entails several important sub-problems. One of these sub-problems is the energy efficiency of the high-power amplifier (HPA) at the very end of the radio frequency chain of the physical layer [1]. The HPA consumes a major fraction of the total energy budget of which, depending on the efficiency class, still much is dissipated as thermal energy and therefore wasted. While smart HPA design can alleviate much of this process there is an intrinsic problem which depends on the form how the HPA is operated: high fluctuations of the input signal-typically quantified by means of the peak-to-average power ratio (PAPR)-enforce operating points typically well below an acceptable efficiency region. This problem becomes particularly serious in multicarrier systems such as OFDM and is the focus of this special issue.

The PAPR problem brings up several challenges for the system designer. One challenge is to adjust HPA design parameters on RF level (which includes pre-distortion techniques as well) in some specific way: here, the tradeoff between power efficiency on the one hand and tolerated nonlinear distortions on the other hand will affect the overall transmission system. It is still unknown how to quantify this effect by a suitable metric. Moreover, the optimal parameters depend on the operational regime set by the signal processing, so that in certain regimes it is better to sacrifice error performance in favor of efficiency and conversely. Popular examples are the EVM (Error Vector Magnitude) metric or the SER (Symbol Error

\footnotetext{
* Correspondence: gerhard.wunder@hhi.fraunhofer.de

${ }^{1}$ Heinrich-Hertz-Institut Berlin, Berlin, Germany

Full list of author information is available at the end of the article
}

Rate) all of them difficult to analyze; Gaussian approximations are helpful but the scope depends on the setting and might be misleading. A second challenge is to process the baseband signal in such a way that the specific metrics are improved: here, the algorithms obviously depend on the choice of the metric. Now, the interaction between both tasks makes it apparent that the PAPR problem involves joint optimization of HPA and the signal processing unit. So far, this interplay has only been marginally addressed in the literature. Figure 1: Joint optimization illustrates this general point of view.

It is a decisive motivation of this special issue that the intrinsic tradeoffs and joint optimization problems are not well understood yet and contain huge potential for future energy efficient design of mobile communication networks. This is particularly true when it comes to multiuser or multi-antenna systems. Illuminating the many aspects of this problem is reflected by the final selection of papers in this special issue which not only includes the latest update of literature but also some new ideas in the direction of fundamental bounds, alternative metrics, joint optimization and multiuser or multi-antenna algorithms for state-of-the-art systems. The editors hope with this special issue to ignite more research related to unsolved questions regarding the important PAPR problem. In the sequel the papers are shortly discussed:

In the paper "PAPR and the Density of Information Bearing Signals in OFDM" by Holger Boche and Brendan Farrell the authors discuss and analyze the value of reserving subcarriers to reduce peak power. The reserved subcarriers approach is one of the simplest and oldest and most well-known approach in literature but at the same time one the analytically hardest to tackle. Hereby, they use a novel approach that analyzes the behavior of metric spaces within the specific structure of OFDM systems. By a nice application of Szemerédi's theorem it is shown then that the efficiency falls below any value if a strict peak power constraint is imposed on the transmission 


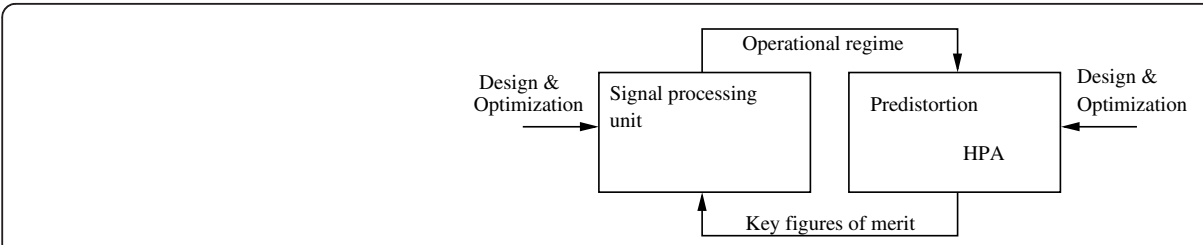

Figure 1 Joint optimization of RF and signal processing.

regardless where the reserved subcarriers are placed. Clearly, these results establish also fundamental bounds on the potential gains due to coding for the PAPR problem.

The analysis of an alternative metric instead of PAPR is the topic of the paper "On the EVM Computation of Arbitrary Clipped Multi-Carrier Signals" by Igal Kotzer and Simon Litsyn. The authors provide exact analytical results for computing the EVM metric circumventing the common Gaussian approximation. The approach uses a novel Fourier representation and power series method to calculate the average distortion energy and it is general enough to capture other HPA models than the standard soft clipping model. The EVM metric is used for evaluation in the paper "Power amplifier linearization technique with IQ imbalance and crosstalk compensation for broadband MIMO OFDM transmitters" by Fernando Gregorio, Juan E. Cousseau, Stefan Werner, Taneli Riihonen, and Risto Wichman where a stochastic control algorithm is devised for a novel joint crosstalk, IQ imbalance and nonlinear amplifier response compensation of a MIMO OFDM transmitter. The authors design a MIMO pre-distortion scheme using a parallel nonlinear model based on memory polynomials where the coefficients are constantly adapted by the underlying control algorithm.

Multiuser downlink systems with multiple antennas are covered in the paper "Selected Basis for PAR Reduction in Multi-User Downlink Scenarios using Lattice-ReductionAided Precoding" by Christian Siegl and Robert Fischer where nonlinear Tomlinson-Harashima-type pre-coding is combined with basis selection algorithm for PAPR reduction. Similarly, in the multiuser MIMO OFDM uplink with DFT pre-coding, the joint problem of maximum throughput and PAPR reduction is tackled in the paper "Sum rate optimization by spatial precoding for a Multiuser MIMO DFT-precoded OFDM uplink" by Hanguang Wu, Thomas Haustein, Eduard Jorswieck, and Peter Hoeher. Here, the idea is to maintain and optimize a frequency non-selective spatial filter in the inner MIMO OFDM system which preserves the excellent PAPR properties of DFT pre-coded OFDM. Adapting the spatial filter is also considered in "Peak-to-Average-Power-Ratio (PAPR) Reduction in WiMAX and OFDM/A Systems" by Seyran Khademi, Thomas Svantesson, Mats Viberg, and
T. Eriksson. Here, a novel approach to finding phase shifts within context of PTS technique is presented.

Space-time-frequency block coded system are analyzed for PAPR reduction in "PAPR Reduction in SFBC MIMO MC-CDMA Systems via User Reservation" by Mariano Garcia Otero and Luis A. Paredes-Hernandez as well as "Low PAPR space frequency block coding for multiuser MIMO SC-FDMA systems: Specific issues for users with different spectral allocations" by Cristina Ciochina, David Mottier, and Damien Castelain. In the first paper a MCCDMA system is addressed where potentially signals of inactive users are added for the benefit of PAPR reduction. In the second paper a DFT pre-coded MIMO OFDM architecture is addressed where a novel space-frequency mapping scheme is devised and analyzed which differs from the standard Alamouti scheme while it preserves low PAPR.

\section{Author details}

${ }^{1}$ Heinrich-Hertz-Institut Berlin, Berlin, Germany ${ }^{2}$ Universität UIm, UIm, Germany ${ }^{3}$ Tel Aviv University, Tel Aviv, Israel ${ }^{4}$ Seoul National University, Seoul, Korea

Received: 24 August 2011 Accepted: 17 September 2011 Published: 17 September 2011

Reference

1. LM Correia, D Zeller, O Blume, D Ferling, Y Jading, I Gdor, G Auer, L Van der Perre, "Challenges and enabling technologies for energy aware mobile radio networks,". IEEE Signal Processing Magazine. 48(11), 66-72 (November 2010)

doi:10.1186/1687-6180-2011-59

Cite this article as: Wunder et al:: Precoding and Transmitter-Side processing techniques for multiuser MIMO OFDM systems with special emphasis on the PAPR problem. EURASIP Journal on Advances in Signal Processing 2011 2011:59. 